\title{
In vivo microvascular imaging of cutaneous actinic keratosis, Bowen's disease and squamous cell carcinoma using dynamic optical coherence tomography
}

\author{
L. Themstrup, ${ }^{1, *}$ G. Pellacani, ${ }^{2}$ J. Welzel, ${ }^{3}$ J. Holmes, ${ }^{4}$ G.B.E. Jemec, ${ }^{1}$ M. Ulrich ${ }^{5}$ \\ ${ }^{1}$ Department of Dermatology, Zealand University Hospital, Roskilde, Denmark \\ ${ }^{2}$ Department of Dermatology, University of Modena and Reggio Emilia, Modena, Italy \\ ${ }^{3}$ Department of Dermatology and Allergology, General Hospital Augsburg, Augsburg, Germany \\ ${ }^{4}$ Michelson Diagnostics Ltd., Kent, UK \\ ${ }^{5} \mathrm{CMB} /$ Collegium Medicum Berlin, Berlin, Germany \\ *Correspondence: L. Themstrup. E-mail: lotte.themstrup@gmail.com
}

\section{Abstract}

Background A clear distinction between actinic keratosis (AK), Bowen's disease (BD) and squamous cell carcinoma (SCC) cannot reliably be made by clinical and dermoscopic evaluation alone. Dynamic optical coherence tomography (D-OCT) is a novel angiographic variant of OCT that allows for non-invasive, in vivo evaluation of the cutaneous microvascular morphology. Objective To investigate the microvascular structures of AK, BD and invasive SCC using D-OCT in order to gain insights into the microvascular morphology of lesions in the spectrum of keratinocyte skin cancers.

Methods Forty-seven patients with a total of 54 lesions (18 AK, 12 BD and 24 SCC) were included in the study. D-OCT still images of $\mathrm{AK}, \mathrm{BD}$ and $\mathrm{SCC}$ at three predefined skin depths were prepared and randomized, creating a study set of 162 D-OCT images. Three observers performed blinded evaluations of the randomized study set assessing multiple parameters including the different types of vascular morphology. Non-blinded quantitative measurements of vascular diameter were also performed.

Results The blinded observer analysis suggests that D-OCT evaluation of the vascular morphology may aid in distinguishing AK, BD and SCC lesions. We identified two vascular shapes that presented significantly differently across the lesion types, namely 'blobs' and 'curves'. A strong presence of blobs at $300 \mu \mathrm{m}$ skin depth was characteristically seen in a third of BD cases, while not or only slightly present in AK and SCC lesions. Vascular curves were predominantly present in AK lesions.

Conclusion We identified various vascular D-OCT features that may aid in non-invasively differentiating subtypes within the keratinocyte skin cancer spectrum.

\section{Conflicts of interest}

Martina Ulrich is stakeholder in CMB Collegium Medicum Berlin GmbH; clinical trials for Almirall, Biofrontera, Leo Pharma and Novartis; paid lectures for Almirall, Biofrontera, Galderma, Leo Pharma, Mavig GmbH, Michelson Diagnostics.

Jon Holmes is Co-founder and Chief Technology Officer at Michelson Diagnostics Ltd.

Gregor B.E. Jemec has received honoraria from AbbVie, MSD and Pfizer for participation on advisory boards, and grants from Abbvie, Actelion, Janssen-Cilag, Leo Pharma, Novartis and Regeneron for participation as an investigator and speaker honoraria from AbbVie, Galderma, Leo Pharma and MSD. He has furthermore received unrestricted research grants from AbbVie, Novartis and Leo Pharma.

Julia Welzel has received speaker honoraria from Almirall, Leo Pharma, Galderma, MSD and Novartis. She received honoraria from Almirall for participation in an advisory board on actinic keratosis.

Giovanni Pellacani has received honoraria from MAVIG and Caliber for participation on advisory boards and teaching and research grants from Abbvie, Leo Pharma, Novartis MSD for clinical studies.

\section{Funding sources}

The project is part of the ADVANCE project that has received funding from the European Union's ICT Policy Support Programme as part of the Competitiveness and Innovation Framework Programme, grant agreement no: 621015. 


\section{Introduction}

In the skin, squamous cell carcinoma (SCC) and actinic keratosis (AK) have important histopathological and aetiological similarities, and the two may be seen as the extremes of a continuous spectrum of neoplastic skin responses to carcinogenic stimuli, most commonly to UV irradiation. ${ }^{1}$ The incidence of actinic keratosis $(\mathrm{AK})$ appears to be increasing and often assumes a chronic course in patients with field cancerization. The patients frequently present with progressive signs of actinic damage, that is increasing numbers of AK's admixed with either Bowen's disease or frank SCC. Patients view AK follow-up with the same apprehension as that of melanoma ${ }^{2}$ which may partially drive the need for diagnosis together with the AK role as being a marker of overall risk of skin cancer development. A clear distinction between AK, Bowen's disease (BD; also termed SCC in situ) and invasive SCC cannot reliably be made by clinical and dermoscopic evaluation alone. Today accurate diagnosis is only possible by histopathological examination of skin biopsies. In consequence, chronic actinic damage poses a significant burden for both patients and society. A need for improved diagnostics therefore exists due to the limited resources. It is hypothesized that in vivo imaging of microvasculature may provide a possible solution.

It is well established that tumours depend on angiogenesis to remain viable and ensure further growth and development, ${ }^{3}$ and the changes that accompany the formation of SCC have been termed the angiogenic switch. ${ }^{4-7}$ Optical coherence tomography (OCT) is a non-invasive tool that allows real-time structural imaging of the skin. Recent technical advances have led to the development of a novel angiographic variation of OCT, named dynamic OCT (D-OCT; a variation of speckle variance). The DOCT technique was recently validated for imaging of skin blood flow $^{8}$ and the first reports on the potential use of D-OCT in diagnosing different skin lesions, including skin cancer, have been published. ${ }^{9-12}$ In continuation of these newly published studies, it is natural to look into D-OCTs ability to differentiate skin cancer subtypes by vascular imaging. The aim of this exploratory study was therefore to investigate the vascular structures of AK, BD and invasive SCC using D-OCT in order to gain insights into the microvascular morphology of lesions in the spectrum of keratinocyte skin cancers.

\section{Materials and Methods}

The study was performed at three European clinical dermatology centres from October 2014 to February 2016. Patients aged $>18$ years with histopathologically verified AK, BD or SCC lesions were included. The institutional review board of each centre approved the study (SJ-408), and all patients provided written informed consent in accordance with the Declaration of Helsinki principles. All lesions were imaged with D-OCT, and in 35 patients, D-OCT images of clinically normal looking adjacent skin were also acquired as controls.

\section{Dynamic OCT}

For D-OCT imaging, we used a commercially available OCT scanner (VivoSight Dx, Michelson Diagnostics, Kent, UK) with a centre wavelength of $1305 \mathrm{~nm}$, an in vivo optical resolution of $<7.5 \mu \mathrm{m}$ lateral; $<5 \mu \mathrm{m}$ axial, an A-Line rate of $20 \mathrm{kHz}$ and a scan area of $6 \times 6 \mathrm{~mm}$. The VivoSight OCT scanner acquires in vivo structural OCT images of the skin to a depth of around $1 \mathrm{~mm}$ and also microcirculation images of the skin to a minimum depth of $500 \mu \mathrm{m}$ (displayed as an overlay). The images are presented in a cross-sectional and an en-face (horizontal) view. The technology behind acquiring in vivo D-OCT images of the microcirculation has previously been described. ${ }^{13}$ For this study, we used a multislice scan modality consisting of $120 \mathrm{~B}$ scans (in the $x-y$ plane) which takes $30 \mathrm{~s}$ to acquire. The scans were collected by experienced D-OCT users, the skin surface was not prepared prior to scanning, and no coupling medium was used. The D-OCT images were acquired by placing the hand-held probe directly on the skin using a fitted plastic spacer for stability and carefully avoiding compression of the skin.

\section{Dynamic OCT image analysis}

In February 2016, all of the authors met to discuss the most suitable way to evaluate vascular features in D-OCT images. The meeting resulted in the identification of six prominent vascular shapes found in D-OCT images of a variety of different lesions and normal skin at different body locations. A short description of the vascular shapes is seen in Fig. 1.

It was further agreed that D-OCT images should be evaluated at three predefined skin depths of 150,300 and $500 \mu \mathrm{m}$ to establish a standard framework for assessing and comparing vascular structures between the different lesions types and normal skin. To view the D-OCT images at fixed depths beneath the skin surface, we utilized the 'Fitted en-face' feature of the VivoSight software (Michelson Diagnostics, Kent, UK). The 'Fitted en-face' tool has previously been described ${ }^{12}$; in short, it detects the skin

\begin{tabular}{ll}
\hline Vascular shapes D-OCT \\
\hline Dots
\end{tabular} $\begin{aligned} & \text { Small red isolated points with a simple round } \\
& \text { appearence } \\
& \text { Blobs }\end{aligned}$

Figure 1 Vascular shapes. Descriptions of the D-OCT vascular shapes identified and used in the blinded observer evaluation. 
surface and allows the imaged skin area $\left(36 \mathrm{~mm}^{2}\right)$ to be viewed at exactly the same depth below the skin surface regardless of skin roughness.

For the purpose of this study, 'Fitted en-face' D-OCT still images of $\mathrm{AK}, \mathrm{BD}$ and SCC were prepared and randomized, creating a study set of 162 D-OCT images. Three of the authors (LT, GJ, MU) performed blinded evaluations of the randomized study set assessing (a) the presence of each vascular shape; (b) the distribution of each vascular shape (absent, regular, irregular, clustered); (c) the predominant vascular shape and (d) the vascular pattern (no pattern, mottle, mesh, cloud, chaos). The presence of each vascular shape was rated on an ordinal scale: (0) not present, (1) present (+), (2) present (++), (3) present $(+++)$. The observers were blinded to diagnosis; clinical appearance, location and imaging depth, and the D-OCT images were presented uniformly on identical screens.

Quantitative measurements of the largest vascular diameter were acquired non-blinded using a build-in measurement tool in the OCT system. Measurements in 35 lesions (12 AK, 6 Bowen, 17 SCC) were compared to D-OCT controls of normal skin adjacent to the lesions. The vascular density was rated on an ordinal scale (less, equal, denser) compared to the corresponding normal adjacent skin at the same skin depth (150, 300, $500 \mu \mathrm{m}$ ). One observer (LT) performed all of the quantitative evaluations.

\section{Statistics}

For analysing the qualitative results of the blinded observer evaluations of vascular morphology, descriptive statistics was applied taking into account the multiple response nature of the data. The results from the evaluation of the vascular shapes from the three observers were analysed separately using $3 \times 4$ contingency tables, layered by skin depth, and Fisher's exact test to test the association between lesion type and the presence of a specific vascular shape. These tests were also performed to test the relationship between lesion type and distribution, predominant shape, pattern and vascular density. A paired-samples $t$-test was used to explore whether there was a difference between the largest vascular diameters in lesional skin compared to normal adjacent skin. For non-normally distributed data, a Wilcoxon signed rank test was performed instead. Lastly, a weighted (quadratic) Light's kappa was performed to measure the interobserver agreement for the three observer evaluations of vascular shapes (categorical data with an ordinal structure). ${ }^{14}$ A $P$-value $\leq 0.05$ was regarded as statistically significant. The analyses were carried out using IBM SPSS Statistics for Macintosh version 22.0 (IBM Corp, Armonk, NY, USA).

\section{Results}

Forty-seven patients (31 males, 16 females) from three European clinical dermatology centres were included in the study. The patients had a total of 54 histopathologically verified lesions consisting of $18 \mathrm{AK}, 12$ Bowen and 24 SCC. Median age was 75 years (range: $39-88$ years), and the lesions were located (number of cases): face (25), nose (3), lip (6), scalp (2), ear (4), trunk (5), arm (4), hand (1), leg (2), foot (1), penis (1).

\section{Qualitative analysis}

The results of the three blinded observer evaluations of vascular shapes at skin depth $300 \mu \mathrm{m}$ are illustrated in Fig. 2.

The results show that at a skin depth of $300 \mu \mathrm{m}$ the presence of vascular blobs differed significantly and consistently across the three diagnoses. All of the observers found that blobs were strongly $(+++)$ present in $33.3 \%$ of BD lesions, while only $(+++)$ present in $4.2-8.3 \%$ of the SCC lesions and not occurring $(+++)$ in any of the AK lesions (illustrated in Fig. 3 and Video $\left.\mathrm{S} 1^{15}\right)$.

Blobs were slightly $(+)$ present in $50-77 \%$ of the AK lesions at depth 300 . At the same skin depth $(300 \mu \mathrm{m})$, two of the observers also found significant differences $(P=0.009$ and $P=0.014$ respectively) in the presence of vascular curves across the three diagnoses. A closer look at the distribution of curves within the lesion types (Fig. 2) showed that all observers found a moderate $(++)$ presence of curves in $28-44 \%$ of AK lesions and that an $a b-$ sence of vascular curves was pronounced in BD lesions (42$58 \%)$ compared to AK (6-28\%) and SCC (25\%) (illustrated in Fig. 3). The observers found no cases of strong $(+++)$ presence of curves at a skin depth of $300 \mu \mathrm{m}$ in any of the diagnoses. The remaining results of the blinded observer evaluation showed that none of the other vascular shapes (dots, coils, lines, serpiginous) presented statistically significant or consistent differences across the diagnoses $\mathrm{AK}, \mathrm{BD}$ and SCC at any of the examined skin depths.

The evaluation of the predominant vascular shape at skin depth $300 \mu \mathrm{m}$ generally showed that dots and blobs were most prevalent in BD lesions and that vascular lines predominated in AK and SCC lesions; however, only one observer found these differences to be significant $(P=0.049)$. At $150 \mu \mathrm{m}$, dots were predominant in all lesion types. The inter-observer agreement for evaluating the vascular shapes showed good agreement regarding vascular lines $(\kappa=0.69)$ and curves $(\kappa=0.63) .{ }^{16}$ Blobs and dots had a moderate agreement, $\kappa=0.60$ and $\kappa=0.47$, respectively, while there was a fair agreement regarding coils $(\kappa=0.32)$ and serpiginous vessels $(\kappa=0.24)$.

Regarding the blinded evaluation of the primary pattern a 'Mottled' pattern predominated in all lesion types at depth $150 \mu \mathrm{m}$. At depth $300 \mu \mathrm{m}$ 'Mesh' was the predominant vascular pattern in AK lesions (16.7-27.8\%), and 'Mottle' was the predominant pattern in $\mathrm{BD}(58.3-75 \%)$, but the differences were not statistically significant. At skin depth $500 \mu \mathrm{m}$ statistically significant differences in the vascular pattern across the lesion types were found by two of the observers, $P=0.04$ and $P=0.004$, respectively. The distribution of the patterns at this depth showed that 'Chaos' was the predominant pattern in SCC 

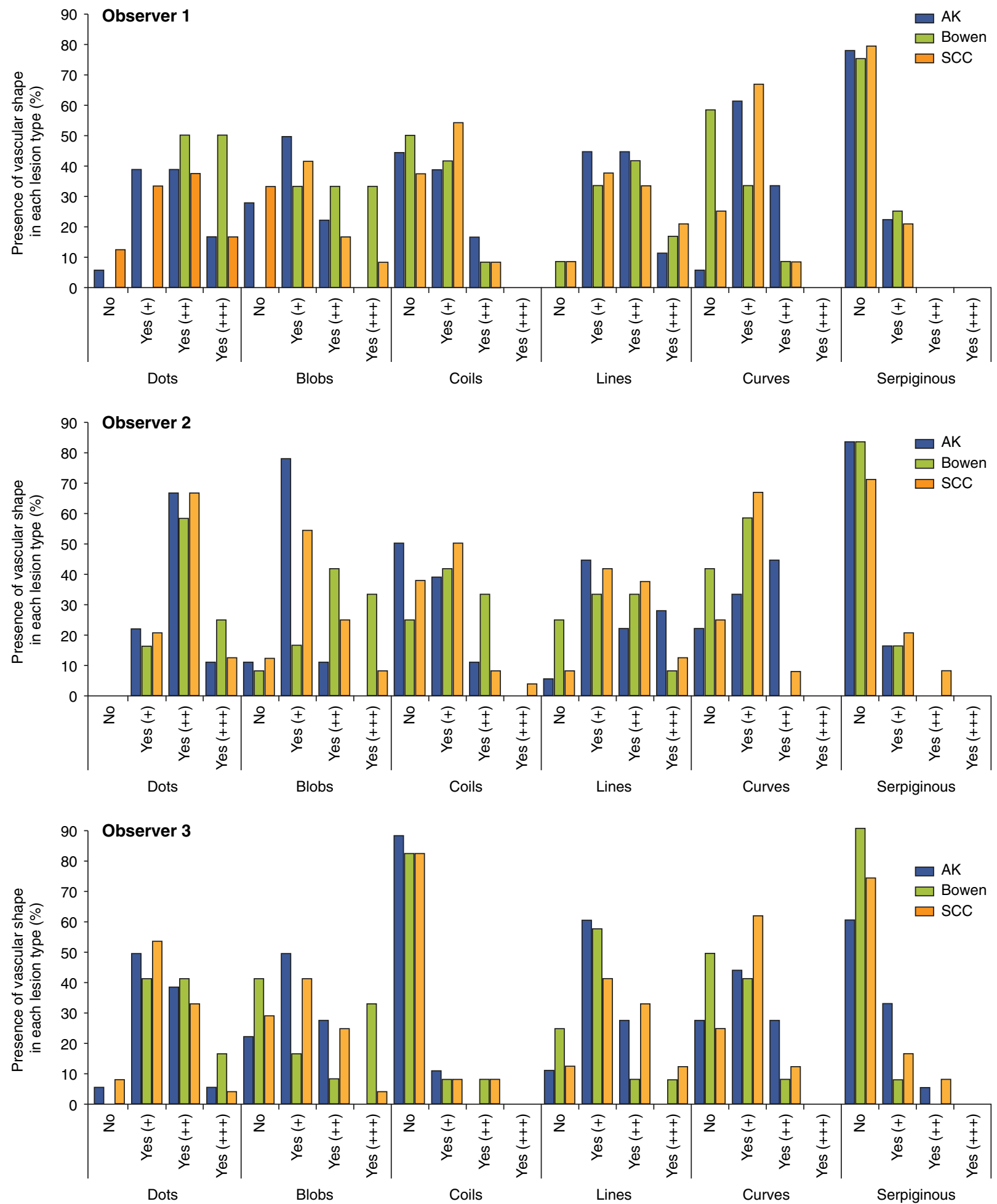

Figure 2 Bar chart illustrating the summarized results of the three blinded observer evaluations of vascular shapes at skin depth $300 \mu \mathrm{m}$. 

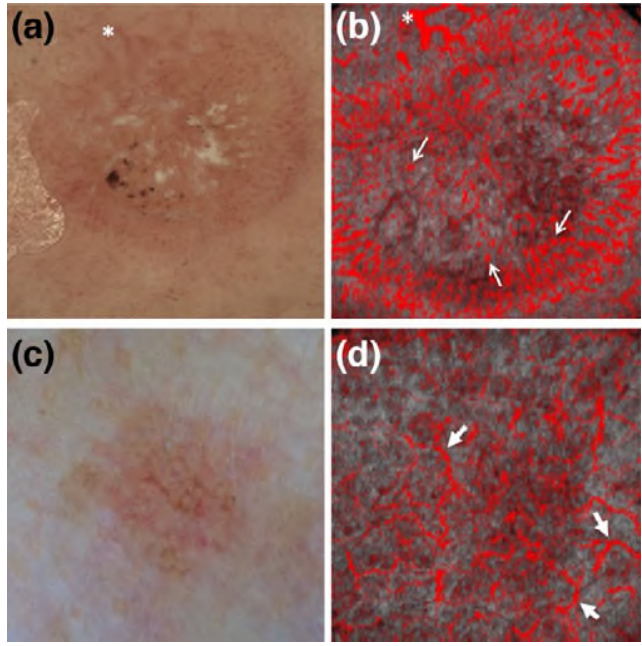

Figure 3 Illustration of vascular blobs and curves. (a) Dermoscopic image (Visiomed AG, Bielefeld, Germany) of a Bowen's disease (BD) lesion located on the cheek. The asterisk marks the exact same vessel seen in image (b). (b) D-OCT image (size: $6 \mathrm{~mm} \times 6 \mathrm{~mm}$; skin depth $300 \mu \mathrm{m}$ ) of the same BD lesion as in (a). The asterisk marks the exact same vessel as in image (a). The thin arrows mark examples of vascular blobs. (c) Dermoscopic image (Handyscope ${ }^{\circledR}$ FotoFinder Systems $\mathrm{GmbH}$, Bad Birnbach, Germany) of an actinic keratosis (AK) located on the face. (d) DOCT image (size $6 \mathrm{~mm} \times 6 \mathrm{~mm}$; skin depth $300 \mu \mathrm{m}$ ) of the same AK lesion as in image (c). The thick arrows mark examples of vascular curves.

(45.8-62.5\%) compared to BD (25.0-33.3\%) and AK (27.8$38.9 \%$ ). Also, the pattern 'Mesh' had a distinct distribution across the three lesion types being present almost exclusively in AK lesions (33.3-38.9\%) compared to Bowen (0\%) and SCC (0$4.2 \%)$. These results are illustrated in Fig. 4. Signs of hyperkeratosis were present in $75-95 \%$ of the images.

\section{Quantitative analysis}

The results of the quantitative measurements of blood vessel diameter are summarized in Table 1 . The quantitative measurements were performed on D-OCT images from 35 patients (12 AK, 6 Bowen and 17 SCC) and compared to reference images consisting of the patient's normal adjacent skin.

The largest vascular diameter found was significantly greater in SCC lesions compared to normal adjacent skin at all skin depths (illustrated in Fig. 4). The results from AK and BD lesions were not significant. A similar tendency was seen in the results from the evaluation of vascular density where SCC lesions consistently had the highest percentage of lesions that fell in the category 'Denser vascularised than the reference image' and the lowest percentage of lesions in the category 'Less vascularised than the reference image' compared to AK and BD (see Fig. 5).
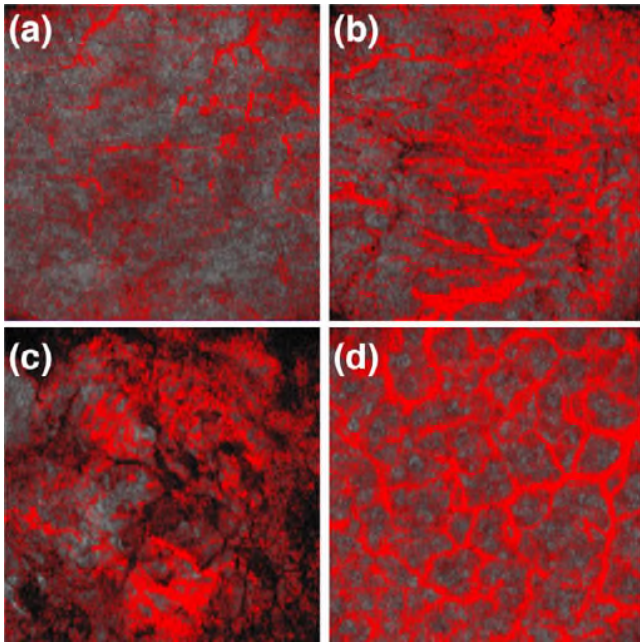

Figure 4 Illustration of vascular diameter and different D-OCT patterns. (a) D-OCT image (size: $6 \mathrm{~mm} \times 6 \mathrm{~mm}$; skin depth $500 \mu \mathrm{m})$ of normal skin on the trunk located adjacent to the squamous cell carcinoma (SCC) lesion seen in image (b). (b) D-OCT image (size: $6 \mathrm{~mm} \times 6 \mathrm{~mm}$ ) of a SCC lesion located on the trunk. The image is taken at the exact same skin depth $(500 \mu \mathrm{m})$ as in (a). Compared to the normal adjacent skin, the vessels here have a larger diameter and appear denser. (c) D-OCT image (size $6 \mathrm{~mm} \times 6 \mathrm{~mm}$; skin depth $500 \mu \mathrm{m}$ ) of a SCC lesion showing a chaotic vascular pattern and dark areas representing hyperkeratosis. (d) D-OCT image (size $6 \mathrm{~mm} \times 6 \mathrm{~mm}$; skin depth $500 \mu \mathrm{m}$ ) of an actinic keratosis lesion showing a characteristic vascular mesh pattern.

The difference between the vascular densities in the three diagnoses was however only statistically significant at depth $150 \mu \mathrm{m}(P=0.01)$. Regrouping the observations into 'Precancer' (AK and BD) and 'Cancer' (SCC) and comparing them did not change the conclusion that the vasculature was generally denser in the 'Cancer' group at all skin depths, however only significantly at the $150 \mu \mathrm{m}$ skin depth $(P=0.016)$.

\section{Discussion}

In this explorative study, we investigated the in vivo microvascular morphology of three lesion types within the keratinocyte skin cancer spectrum by using D-OCT. The results of our blinded observer analysis suggest that D-OCT evaluation of the vascular morphology may help distinguish the lesion types in this spectrum. We identified two vascular shapes that differed across the lesions types: blobs and curves (see Fig. 3 and Video $S 1^{15}$ ). The vascular shape blobs were strongly present in one-third of the evaluated $\mathrm{BD}$ cases at a skin depth of $300 \mu \mathrm{m}$, while none of the AK lesions displayed a strong presence of blobs and only few per cent of the SCC lesions presented this characteristic vascular shape. This finding corresponds well with previous dermoscopy studies on keratinocyte skin cancer where the presence of 
Table 1 Blood vessel diameter. Summary of the results of the quantitative measurements of blood vessel diameter. A paired-samples $t$ test was used for evaluating the difference between lesional and normal skin

\begin{tabular}{|c|c|c|c|c|c|c|}
\hline Skin depth & $n$ & $\begin{array}{l}\text { Mean vascular } \\
\text { diameter }(\mathrm{mm}) \\
\text { Lesional skin }\end{array}$ & $\begin{array}{l}\text { Mean vascular } \\
\text { diameter }(\mathrm{mm}) \\
\text { Adjacent normal skin }\end{array}$ & Difference & $\begin{array}{l}95 \% \text { confidence } \\
\text { interval }\end{array}$ & $P$-value \\
\hline \multicolumn{7}{|l|}{ Actinic keratosis } \\
\hline All skin depths combined & 36 & 0.114 & 0.108 & 0.006 & -0.015 to 0.026 & 0.6 \\
\hline 150 & 12 & 0.074 & 0.052 & 0.022 & -0.004 to 0.047 & 0.097 \\
\hline 300 & 12 & 0.127 & 0.119 & 0.008 & -0.024 to 0.041 & 0.6 \\
\hline 500 & 12 & $0.13 \dagger$ & $0.15 \dagger$ & -0.02 & - & 0.47 * \\
\hline \multicolumn{7}{|l|}{ Bowen's disease } \\
\hline All skin depths combined & 18 & $0.093 \dagger$ & $0.075 \dagger$ & 0.018 & - & $0.04^{*}$ \\
\hline 150 & 6 & $0.068 \dagger$ & $0.035 \dagger$ & 0.033 & - & $0.094^{\star}$ \\
\hline 300 & 6 & $0.095 \dagger$ & $0.089 \dagger$ & 0.006 & - & $0.313^{*}$ \\
\hline 500 & 6 & $0.117 \dagger$ & $0.1 \dagger$ & 0.017 & - & $0.625^{*}$ \\
\hline \multicolumn{7}{|l|}{ Squamous cell carcinoma } \\
\hline All skin depths combined & 51 & 0.13 & 0.09 & 0.04 & 0.027 to 0.058 & $<0.0005$ \\
\hline 150 & 17 & $0.07 \dagger$ & $0.03 \dagger$ & 0.04 & - & $<0.0005^{*}$ \\
\hline 300 & 17 & 0.15 & 0.1 & 0.05 & 0.001 to 0.077 & 0.015 \\
\hline 500 & 17 & 0.18 & 0.14 & 0.04 & 0.011 to 0.069 & 0.011 \\
\hline
\end{tabular}

*Non-normally distributed data, Wilcoxon Signed Rank test used.

$\dagger$ †edian reported.

glomerular vessels, often arranged in clusters, was found to have a positive predictive value of $61.9 \%$ for $\mathrm{BD}$ and only $4.8 \%$ for SCC. ${ }^{17,18}$ Although none of the observers in our study found that the vascular blobs were distributed in clusters, the dermoscopic description of glomerular vessels as 'Larger calibre reddish dots $^{\prime 19}$ seems consistent with our description of blobs in DOCT. Thus, the combination of structural OCT changes previously described in $\mathrm{BD}^{20,21}$ and the pronounced presence of vascular blobs may be a valuable D-OCT clue in differentiating BD from AK and SCC at a skin depth of $300 \mu \mathrm{m}$. The same applies for vascular curves. We found vascular curves to be moderately present in up to $44 \%$ of the of AK lesions, while only moderately

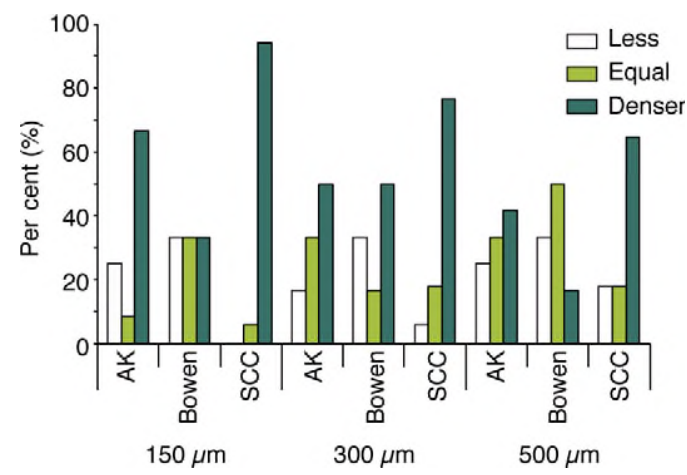

Figure 5 Bar chart illustrating the summarized results from the evaluation of vascular density at all skin depths $(150,300$, $500 \mu \mathrm{m})$. present in $8.3 \%$ of $\mathrm{BD}$ lesions and up to $12.3 \%$ of the SCC lesions. Furthermore, vascular curves were totally absent in up to $58 \%$ of the BD lesions. A moderate presence of curved vessels in lesions at a $300 \mu \mathrm{m}$ skin depth may therefore indicate that the lesion is precancerous with only mild to moderate epidermal dysplasia. None of the other vascular shapes (dots, coils, lines, serpiginous) presented significant differences across the three lesion types at any skin depth. This negative finding is supported by previous dermoscopy studies. The presence of coiled vessels in dermoscopy is described as unhelpful when differentiating keratoacanthoma, SCC, AK and BD because coiled vessels are commonly found in all of these lesions. ${ }^{22}$ Contrary to globular vessel, that are dermoscopically reported only in $\mathrm{BD}$, the 'classical' dotted vessels have been described as being frequently seen in a variety of non-pigmented skin tumours. ${ }^{23}$

The specific vascular pattern might also provide diagnostic clues for differentiating the keratinocyte skin cancer subtypes. Overall, the blinded analysis revealed that the vascular pattern differed significantly across the diagnoses at depth $500 \mu \mathrm{m}$ and that a chaotic pattern predominated in SCC lesions while a welldefined mesh-like pattern was present almost exclusively in onethird of the AK lesions (Fig. 3). These D-OCT results correspond well with previous dermoscopic findings where a polymorphous vascular pattern has been associated with poorly differentiated SCC, while a red pseudonetwork resembling that of normal skin has been described as a feature in early $\mathrm{AK}$ lesions. ${ }^{19,24,25}$ This also supports the notion that a stepwise pattern of angiogenesis evolves during tumour development starting in early AK with vascular features similar to the surrounding 
normal skin, then dotted or glomerular vessels (orientated vertically to the skin surface) and finally progressing to elongated, linear vessels (orientated horizontally to the skin surface) and a polymorphous pattern in SCC lesions. ${ }^{7,24,26}$

Our quantitative measurements of largest vascular diameter showed that only SCC lesions had a significantly larger vascular diameter compared to normal skin. The D-OCT vascular density was also found to be greater in SCC lesions. These results fit well with the above-mentioned microvascular progression model in keratinocyte skin cancers, and previous histological ex vivo studies have also demonstrated increased vessel size as a predominant response in late stage SCC. ${ }^{3,27}$

As mentioned above, our results correspond well with previous findings in dermoscopy and histology studies. Compared to histology, both dermoscopy and D-OCT allows for in vivo evaluation of the vascular morphology in a quick and non-invasive way. D-OCT provides high-resolution microvascular imaging to a greater skin depth than dermoscopy. In dermoscopy, upper dermal vessels appear pink and out of focus due to dispersion of light through the connective tissue. ${ }^{19} \mathrm{D}$-OCT imaging provides clear images of the microvasculature with a resolution high enough to detect small capillaries (diameter $>10 \mu \mathrm{m}$ ) combined with information on skin depth. This allows for detailed in vivo examination of the vascular morphology, although the full potential benefits of D-OCT remain to be explored.

\section{Limitations}

The agreement on the vascular morphology between the three observers varied from $\kappa=0.24$ to $\kappa=0.69$ depending on which specific vascular shape was evaluated. The poor agreement on some of the vascular shapes emphasizes the need to develop and disseminate clearly stated guidelines for rendering the evaluations of the D-OCT images. Because of the explorative nature of this study, some disagreement was expected; however, future DOCT studies relying on observer evaluations may benefit from guideline-based training sessions preceding the evaluations. Regarding the quantitative measurements, our results were limited by only having six BD cases comparable to normal adjacent skin, furthermore only one of the BD cases were located on nonsun-exposed skin. This may have influenced our results, and further D-OCT studies on BD lesions are warranted. The technical limitations of D-OCT have previously been described. ${ }^{8,12}$

\section{Conclusion}

In this explorative study, we identified various vascular D-OCT features that may aid in non-invasively differentiating subtypes within the keratinocyte skin cancer spectrum. D-OCT opens up new possibilities for detailed in vivo exploration of the cutaneous microvascular morphology and may refine our understanding of the biological principles underpinning skin cancer development. Future studies on the diagnostic accuracy of D-OCT in diagnosing different types of skin cancers are warranted.

\section{Acknowledgements}

The project is part of the ADVANCE project that has received funding from the European Union's ICT Policy Support Programme as part of the Competitiveness and Innovation Framework Programme, grant agreement no: 621015. The manuscript reflects only the author's views, and the European Union is not liable for any use that might be made of information contained herein. We are also grateful to Jørgen Lock-Andersen, Consultant, DMSc at Department of Plastic surgery; Zealand University Hospital for referring patients to our study and to Meike Homan at CMB and Silvana Ciardo at UNIMORE for helping with data collection. We would like to thank Adam Andersen Læssøe, MSc, for randomizing and derandomizing data from the study. We thank the Section of Biostatistics at Copenhagen University for statistical advice and Richard Whitehead, for developing software that aided the OCT data analysis. We also thank Marco Manfredi for developing software that allows the D-OCT images to be presented in $3 \mathrm{D}$ view.

\section{References}

1 Dirschka T, Gupta G, Micali G et al. Real-world approach to actinic keratosis management: practical treatment algorithm for office-based dermatology. J Dermatolog Treat 2016; 1-12.

2 Themstrup L, Jemec GE, Lock-Andersen J. Patients highly value routine follow-up of skin cancer and cutaneous melanoma. Dan Med J 2013; 60: A4713.

3 Bolontrade MF, Stern MC, Binder RL, Zenklusen JC, Gimenez-Conti IB, Conti CJ. Angiogenesis is an early event in the development of chemically induced skin tumors. Carcinogenesis 1998; 19: 2107-2113.

4 Folkman J, Watson K, Ingber D, Hanahan D. Induction of angiogenesis during the transition from hyperplasia to neoplasia. Nature 1989; 339 58-61.

5 Strieth S, Hartschuh W, Pilz L, Fusenig NE. Angiogenic switch occurs late in squamous cell carcinomas of human skin. Br J Cancer 2000; 82: 591600 .

6 Johnson KE, Wilgus TA. Multiple roles for VEGF in non-melanoma skin cancer: angiogenesis and beyond. J Skin Cancer 2012; 2012: 483439.

7 Tehrani H, McArthur P, Dalal M. Visualizing the vascular history of nonmelanoma skin tumors: an in vivo human study. Ann Plast Surg 2013; 70: 717-719.

8 Themstrup L, Welzel J, Ciardo S et al. Validation of Dynamic optical coherence tomography for non-invasive, in vivo microcirculation imaging of the skin. Microvasc Res 2016; 107: 97-105.

9 Ulrich M, Themstrup L, de Carvalho N et al. Dynamic optical coherence tomography in dermatology. Dermatology 2016; 232: 298-311.

10 Markowitz O, Schwartz M, Minhas S, Siegel DM. Speckle-variance optical coherence tomography: a novel approach to skin cancer characterization using vascular patterns. Dermatol Online J 2016; 22. PMID: 27617454.

11 De Carvalho N, Ciardo S, Cesinaro A et al. In vivo micro-angiography by means of speckle-variance optical coherence tomography (SV-OCT) is able to detect microscopic vascular changes in naevus to melanoma transition. J Eur Acad Dermatol Venereol 2016; 30: e67-e68.

12 Themstrup L, Ciardo S, Manfredi M et al. In vivo, micro-morphological vascular changes induced by topical brimonidine studied by Dynamic optical coherence tomography. J Eur Acad Dermatol Venereol 2016; 30: 974-979.

13 Ulrich M, Themstrup L, de Carvalho $\mathrm{N}$ et al. Dynamic optical coherence tomography in dermatology. Dermatology 2016; 232: 298-311. 
14 Hallgren KA. Computing inter-rater reliability for observational data: an overview and tutorial. Tutor Quant Methods Psychol 2012; 8: 23-34.

15 Manfredi M, Grana C, Pellacani G. Skin surface reconstruction and 3D vessels segmentation in speckle variance optical coherence tomography. Proceedings of the 11th Joint Conference on Computer Vision, Imaging and Computer Graphics Theory and Applications. 2016; 4.

16 Landis JR, Koch GG. The measurement of observer agreement for categorical data. Biometrics 1977; 33: 159-174.

17 Zalaudek I, Argenziano G, Leinweber B et al. Dermoscopy of Bowen's disease. Br J Dermatol 2004; 150: 1112-1116.

18 Argenziano G, Zalaudek I, Corona R et al. Vascular structures in skin tumors: a dermoscopy study. Arch Dermatol 2004; 140: 1485-1489.

19 Martin JM, Bella-Navarro R, Jorda E. Vascular patterns in dermoscopy. Actas Dermosifiliogr 2012; 103: 357-375.

20 Schmitz L, Bierhoff E, Dirschka T. Optical coherence tomography imaging of erythroplasia of Queyrat and treatment with imiquimod 5\% cream: a case report. Dermatology 2014; 228: 24-26.

21 Forsea AM, Carstea EM, Ghervase L, Giurcaneanu C, Pavelescu G. Clinical application of optical coherence tomography for the imaging of nonmelanocytic cutaneous tumors: a pilot multi-modal study. J Med Life 2010; 3: 381-389.

22 Rosendahl C, Cameron A, Argenziano G, Zalaudek I, Tschandl P, Kittler H. Dermoscopy of squamous cell carcinoma and keratoacanthoma. Arch Dermatol 2012; 148: 1386-1392.
23 Zalaudek I, Di Stefani A, Argenziano G. The specific dermoscopic criteria of Bowen's disease. J Eur Acad Dermatol Venereol 2006; 20: 361-362.

24 Zalaudek I, Argenziano G. Dermoscopy of actinic keratosis, intraepidermal carcinoma and squamous cell carcinoma. Curr Probl Dermatol 2015; 46: 70-76.

25 Peris K, Micantonio T, Piccolo D, Fargnoli MC. Dermoscopic features of actinic keratosis. J Dtsch Dermatol Ges 2007; 5: 970-976.

26 Zalaudek I, Giacomel J, Schmid K et al. Dermatoscopy of facial actinic keratosis, intraepidermal carcinoma, and invasive squamous cell carcinoma: a progression model. J Am Acad Dermatol 2012; 66: 589-597.

27 Chin CW, Foss AJ, Stevens A, Lowe J. Differences in the vascular patterns of basal and squamous cell skin carcinomas explain their differences in clinical behaviour. J Pathol 2003; 200: 308-313.

\section{Supporting information}

Additional Supporting Information may be found in the online version of this article:

Video S1. 3D video of the D-OCT in vivo microvasculature of a Bowen's disease lesion (MPEG, $22 \mathrm{MB}$ ) 